\section{IN VIVO THERAPEUTIC SUCCESS OF MICRORNA-155 (MIR-155) ANTAGOMIR IN A MOUSE MODEL OF LUPUS ALVEOLAR HAEMORRHAGE}

Y Wang*, S Zhou, N Shen. RenJi Hospital- School of Medicine- Shanghai JiaoTong University, Department of Rheumatology, Shanghai, China

\subsection{6/lupus-2017-000215.333}

Background and aims Diffuse alveolar haemorrhage (DAH) is a rare but life-threatening complication of systemic lupus erythematosus (SLE). Pristane-treated B6 mice develop severe DAH within 2 weeks of treatment. MicroRNA-155 (miR-155) is a pleiotropic microRNA that plays a crucial role in the regulation of immune responses. The purpose of this study was to examine the role of miR-155 in the development of DAH in pristane-induced lupus using miR-155-knockout (miR-155-/-) mice and miR-155 antagomir to silence miR-155.

Methods DAH was induced by an intraperitoneal injection of $0.5 \mathrm{~mL}$ of pristane. MiR-155 antagomir was intravenously administrated to silence miR-155 expression. Lung tissues were collected for RNA extraction and were embedded in paraffin for sectioning. Gene expression profiling data were analysed using Ingenuity Pathway Analysis. Real time q-PCR was used for single validation. Luciferase reporter assay and RNAAgo2 immunoprecipitation were performed for target validation.

Results MiR-155 expression was significantly increased in the development of DAH. Disease progression was reduced in miR-155/- mice and by in vivo silencing of miR-155 using miR-155 antagomir. MiR-155 silencing dampened pristaneinduced ectopic activation of multiple inflammatory pathways, and reduced the expression of pro-inflammatory cytokines. Several negative regulators of nuclear factor (NF)- $\mathrm{\kappa B}$ signalling

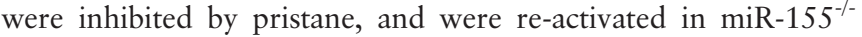
mice. In particular, the anti-inflammatory factor peroxisome proliferator-activated receptor- $\alpha$ was identified as a direct target of miR-155.

Conclusions MiR-155 promotes pristane-induced lung inflammation. MiR-155 contributes to ectopic activation of NF- $\kappa \mathrm{B}$ signalling pathways by targeting multiple negative regulators. MiR-155 antagomir may be a promising therapeutic strategy for treating acute lung inflammation in lupus.

\section{SERUM IL-18 AS BIOMARKER IN PREDICTING LONG TERM RENAL OUTCOME AMONG PEDIATRIC-ONSET SYSTEMIC LUPUS ERYTHEMATOSUS PATIENTS}

JL ${ }^{1} \mathrm{KW}$ Yeh*, 'CY Wu, ${ }^{2} \mathrm{HY}$ Yang, ${ }^{1} \mathrm{~J} \mathrm{~L} .{ }^{1}$ Chang Gung Memorial Hospital, Paediatrics, Taoyuan, Taiwan R.O.C; ${ }^{2}$ Chang Gung Memorial Hospital, Nephrology, Taoyuan, Taiwan R. O.C

\subsection{6/lupus-2017-000215.334}

Background and Aims An urge of biomarker identification is needed to better monitor lupus nephritis (LN) disease activity, guide clinical treatment, and predict patient's long-term outcome. With the proinflammatory effect and its association with inflammasomes, the significance of nterleukin-18 (IL-18) among pediatric-onset systemic lupus erythematous (pSLE) patient.

Methods In a pSLE cohort of 96 patients with an average follow-up period of $10.39 \pm 3.31$ years, clinical data and laboratory workups including serum IL-18 were collected at time of disease onset and 6 months after treatment despite their initial renal status. Through Cox regression analysis, the parameters at baseline and at 6 months posttreatment were carefully analysed.

Results Average age of all cases was 12.74 \pm 3.01 years old and 65 of them underwent renal biopsy at the time of diagnosis. Nine $(9.38 \%)$ progressed to end-stage renal disease (ESRD) and $2(2.08 \%)$ died during follow-up. Through multivariate analysis, serum IL-18 level 6 months posttreatment was found to be the most unfavourable factor associating poor clinical outcome despite patient's initial renal status. The presentation of serum IL-18 in its correlation with SLE global disease activity as well as the presence and severity of LN were all significant $(p<0.001, p=0.03$, and $p=0.02$, respectively). The histological classification of $\mathrm{LN}$ was not associated with the level of IL-18 among the pSLE patients $(p=0.64)$. Conclusions The role of serum IL-18 as biomarker representing global disease activity and status of renal flares among pSLE population was shown for the first time. Additionally, we have identified IL-18 at 6 months posttreatment a novel marker for long-term renal outcome prediction.

\section{MICRORNA-21 IS A CRITICAL REGULATOR OF AUTOIMMUNITY THROUGH PROMOTING EFFECTOR AND METABOLIC FUNCTION OF PATHOGENIC TH17 CELLS}

X Yu*, D Dai, N Shen. RenJi Hospital- School of Medicine- Shanghai JiaoTong University, Department of Rheumatology, Shanghai, China

\subsection{6/lupus-2017-000215.335}

Background and aims Systemic lupus erythematosus is a prototypical autoimmune disease that causes mortality and morbidity worldwide. Recent studies suggest proinflammatory TH17 cells are key pathogenic factors that contribute to lupus nephritis. Our group previously demonstrate that microRNA-21 was highly upregulated in $\mathrm{CD}^{+} \mathrm{T}$ cells from both lupus patients and lupus-prone mice. However, the role of microRNA-21 in pathogenic TH17 cells and they-mediated autoimmune diseases is still unclear. In this study, we systemically dissect the role of microRNA-21 in the differentiation and effector function of pathogenic TH17 cells.

Methods MicroRNA-21 knockout and conditional knockout mice were generated. EAE was induced to study the role of microRNA-21 in pathogenic TH17 cell-mediated autoimmune diseases. RNA-seq, RIP-seq and DAVID bioinformatic analysis were conducted to find key microRNA-21 regulated pathway and molecular targets in pathogenic TH17 cells. Metabolic assays were done to study the glycolytic activity of microRNA-21-deficent pathogenic TH17 cells.

Results In this study, we demonstrate that IL-6-STAT3 signalling induced microRNA-21 is essential for the late stage commitment and maintenance of pathogenic TH17 cells by targeting key regulators. MicroRNA-21-deficient TH17 cells express less pathogenic TH17 signature genes and show less glycolytic activity. Conditional deletion of microRNA-21 in $\mathrm{CD}^{+}{ }^{+} \mathrm{T}$ cells protects mice from EAE while loss of microRNA-21 expression by dendritic cells and myeloid cells do not.

Conclusions These findings suggest that microRNA-21 is a novel cell-intrinsic regulator of the commitment and metabolic function of pathogenic TH17 cells. It may be a potential therapeutic candidate with which to reprogram the immune system and help prevent and treat autoimmune diseases. 